\title{
On Decay-nondecay and Scattering for Schrödinger Equations with Time Dependent Complex Potentials
}

\author{
By
}

Kiyoshi Mochizuki* and Takahiro MotaI**

\begin{abstract}
We consider the Schrödinger equations with time dependent complex potentials. Under suitable space-time decaying conditions on the potential we treat $L^{2}$ decaynondecay of solutions and also develop a scattering theory.
\end{abstract}

\section{$\S 1 . \quad$ Introduction}

We consider the Schrödinger equation

$$
i \partial_{t} u-\Delta u+V(x, t) u=0, \quad(x, t) \in \mathbf{R}^{n} \times \mathbf{R},
$$

where $i=\sqrt{-1}, \partial_{t}=\partial / \partial t, \Delta$ is the $n$-dimensional Laplacian and $V(x, t)$ is a complex potential which is bounded and continuous in $\mathbf{R}^{n} \times \mathbf{R}$.

We choose the initial condition at $t=0$,

$$
u(x, 0)=f(x) \in L^{2},
$$

and restrict ourselves to solutions in $L^{2}$. Here, for $0 \leq p \leq \infty, L^{p}=L^{p}\left(\mathbf{R}^{n}\right)$ is the usual $L^{p}$-space with norm

$$
\|f\|_{L^{p}}=\left\{\int_{\mathbf{R}^{n}}|f(x)|^{p} d x\right\}^{1 / p}(1 \leq p<\infty), \quad\|f\|_{L^{\infty}}=\operatorname{ess} \sup _{x \in \mathbf{R}^{n}}|f(x)| .
$$

Communicated by T. Kawai. Reseived July 31, 2006. Revised February 23, 2007. 2000 Mathematics Subject Classification(s): Primary 35P25; Secondary 35B40.

*Faculty of Science and Engineering, Chuo University, Kasuga, Bunkyo-ku, Tokyo 1128551, Japan.

e-mail: mochizuk@math.chuo-u.ac.jp

** Japanese Language Center for International Students, Tokyo University of Foreign Studies, Sumiyoshi, Fuchu, Tokyo 183-0034, Japan.

e-mail: motai@tufs.ac.jp

(c) 2007 Research Institute for Mathematical Sciences, Kyoto University. All rights reserved. 
In the following we simply write $\int_{\mathbf{R}^{n}}=\int$ and omit the suffix $L^{2}$ of $\|\cdot\|_{L^{2}}$ when $p=2$.

Let $U_{0}(t)=e^{-i t \Delta}$ be the unitary group in $L^{2}$ which represents the solution of the free equation

$$
i \partial_{t} u_{0}-\Delta u_{0}=0
$$

Then problem (1), (2) reduces to the integral equation

$$
u(t)=U_{0}(t) f+i \int_{0}^{t} U_{0}(t-\tau) V(\cdot, \tau) u(\tau) d \tau .
$$

For given $f \in L^{2}$, this equation has a unique solution $u(t) \in C\left(\mathbf{R} ; L^{2}\right)$. We denote by $U(t, s) \in \mathcal{B}\left(L^{2}\right)$ the evolution operator which maps solutions at time $s$ to those at time $t$ :

$$
u(t)=U(t, s) u(s) .
$$

The unique existence of solutions of (4) implies that for each fixed $s$ and $t$, $U(t, s)$ defines a bijection on $L^{2}$.

In this paper, under suitable conditions on $V(x, t)$, we shall treat decaynondecay of solutions, and develop a scattering theory.

As is easily seen (Lemma 1 (i)), we have

$$
\|u(t)\|^{2}+\int_{0}^{t} \int \operatorname{Im} V(x, t)|u(x, \tau)|^{2} d x d \tau=\|u(s)\|^{2}
$$

for any $t>0$, where $\operatorname{Im} V(x, t)$ denotes the imaginary part of $V(x, t)$. If $\operatorname{Im} V(x, t) \geq 0$, then $\|u(t)\|$ is decreasing with $t$, and a question rises whether it decays or not as $t$ goes to infinity.

The decay-nondecay problems of solutions have been studied for dissipative wave equations (see e.g. Mochizuki-Nakazawa [11]) based on the energy identity corresponding to (5) and a space-time weighted energy estimate of free solutions. In case of the Schrödinger equation, we can follow a similar line of proof if the last estimate is replaced by the so-called $L^{p}-L^{q}$ estimates of free solutions.

The scattering theory compares solutions of (1) and (3) not only when $t \rightarrow \infty$ but also when $t \rightarrow-\infty$. So, the positivity of $\operatorname{Im} V(x, t)$ in $(5)$ does not work well, and it is necessary to obtain convenient space time estimates of perturbed solutions. There are several works which treat time dependent potentials. See Howland [2], Yafaev [12], Yajima [13], Kitada-Yajima [7] and Jensen [3]. But their results are restricted to the case of real potentials. So, for each fixed $t$ the operator $-\Delta+V(x, t)$ becomes selfadjoint, and this fact plays 
an important role in their theory. In this paper, in place of the selfadjointness, we require a smallness condition on $V(x, t)$.

For time independent complex potentials, the smooth pertubation theory has been developed by Kato's classical paper [4] (see also Kato-Yajima [5] and Mochizuki [8]) to treat small perturbations. This theory is based on the weighted resolvent estimate, and is not available either in our time dependent potential. In this paper, by solving the integral equation (4), we directly obtain a necessary $L^{p}-L^{q}$ estimate for perturbed problem (1). Note that in the recent work of Mochizuki [9] the corresponding results on scattering have been shown for wave equations with time dependent coefficient, where is used a space time weighted energy estimate of pertubed solutions.

Now, let us explain the results of this paper for a typical example

$$
V(x, t)=c(1+r)^{-\alpha}(1+|t|)^{-\beta} \quad(r=|x|)
$$

with $c \in \mathbf{C}$ and $\alpha, \beta \geq 0$.

In the next Section 2 we shall first show (Theorem 1) that $L^{2}$ decay $\|u(t)\| \rightarrow 0(t \rightarrow \infty)$ occurs if we require

$$
\operatorname{Im} c>0 \text { and } \quad \alpha+\beta \leq 1
$$

Contrary to this condition, if we require

$$
\operatorname{Im} c>0 \text { and } \quad \alpha+\beta>1,
$$

then as will be seen (Theorem 2) $\|u(t)\|$ does not in general decay as $t \rightarrow \infty$.

In Section 3 we shall obtain space-time $L^{p}-L^{q}$ estimates of $u(t)$ (Theorem 3) based on similar estimates of free solutions. For this aim, we restrict ourselves to complex potentials like

$$
\frac{\alpha}{2}+\beta>1 \text { and }|c| \text { is small if } \beta=0 .
$$

Finally, in Section 4 these estimates are used to develop a scattering theory (Theorem 4). As will be shown, the strong limit

$$
Z^{ \pm}=s-\lim _{t \rightarrow \pm \infty} U_{0}(-t) U(t, 0)
$$

exists under (9). Moreover, it gives a bijection on $L^{2}$ if $|c|$ in (9) is restricted smaller. In this case the $\mathrm{M} \phi$ ller wave operator is obtained by $W^{ \pm}=\left(Z^{ \pm}\right)^{-1}$ and the scattering operator is defined as follows:

$$
S=\left(W^{+}\right)^{-1} W^{-}=Z^{+}\left(Z^{-}\right)^{-1} .
$$


Note that example (6) has been given in Yafaev [12] when $c$ is real and $\beta>0$. His results include the following. The wave operator

$$
W^{ \pm}=s-\lim _{t \rightarrow \pm \infty} U(0, t) U_{0}(t)
$$

exists if $\alpha+\beta>1$. It is in general incomplete, but becomes complete, i.e., the range of $W^{ \pm}$coincides with the whole space $L^{2}$, if the stronger condition $\frac{\alpha}{2}+\beta>1$ is required.

\section{$\S 2 . \quad L^{2}$ Decay and Nondecay of Solutions}

In the following we distinguish the real and imaginary parts of $V(x, t)$ by $V_{R}(x, t)$ and $V_{I}(x, t)$, respectively:

$$
V(x, t)=V_{R}(x, t)+i V_{I}(x, t) .
$$

Lemma 1. Let $u(t)$ be the $L^{2}$ solution of (1), (2).

(i) Assume that $V(x, t)$ is bounded, continuous in $\mathbf{R}^{n} \times \mathbf{R}$. Then we have

$$
\frac{1}{2}\|u(t)\|^{2}+\int_{0}^{t} \int V_{I}(x, \tau)|u|^{2} d x d \tau=\frac{1}{2}\|f\|^{2} .
$$

(ii) Assume further that $\partial_{t} V_{R}(x, t)$ and $\nabla V_{I}(x, t)$ are bounded, continuous in $\mathbf{R}^{n} \times \mathbf{R}$. Then we have

$$
\begin{gathered}
\left.\frac{1}{2} \int\left\{|\nabla u|^{2}+V_{R}(x, t)|u|^{2}\right\} d x\right|_{0} ^{t}+\int_{0}^{t} \int\left[V_{I}(x, t)\left\{|\nabla u|^{2}+V_{R}(x, t)|u|^{2}\right\}\right. \\
\left.+\operatorname{Re}\left\{\left(\nabla V_{I}(x, t) \cdot \nabla u\right) \bar{u}\right\}-\frac{1}{2} \partial_{t} V_{R}(x, t)|u|^{2}\right] d x d t=0
\end{gathered}
$$

Proof. By a standard approximation procedure (see Remark given below), we have only to show these identities for smooth $u(t) \in C\left((0, \infty) ; H^{2}\right) \cap$ $C^{1}\left((0, \infty) ; L^{2}\right)$. Here $H^{j}(j=1,2)$ is the Sobolev space with norm

$$
\|f\|_{H^{j}}^{2}=\int\left\{|f(x)|^{2}+\left|\nabla^{j} u\right|^{2}\right\} d x<\infty .
$$

(i) We multiply by $\bar{u}$ on both sides of (1). Then

$$
i u_{t} \bar{u}-\nabla \cdot\{(\nabla u) \bar{u}\}+|\nabla u|^{2}+V(x, t)|u|^{2}=0,
$$

where $u_{t}=\partial_{t} u$. Taking the imaginary parts, we have

$$
\frac{1}{2} \partial_{t}|u|^{2}-\operatorname{Im}[\nabla \cdot\{(\nabla u) \bar{u}\}]+V_{I}(x, t)|u|^{2}=0 .
$$


Integration by parts on $\mathbf{R}^{n} \times(0, t)$ then gives the desired identity.

(ii) We take the real parts of (10) and multiply both sides by $V_{I}(x, t)$. Then

$$
\begin{gathered}
-V_{I}(x, t) \operatorname{Im}\left(u_{t} \bar{u}\right)-\operatorname{Re}\left[\nabla \cdot\left\{V_{I}(x, t)(\nabla u) \bar{u}\right\}\right]+\operatorname{Re}\left[\left\{\nabla V_{I}(x, t) \cdot \nabla u\right\} \bar{u}\right] \\
+V_{I}(x, t)\left\{|\nabla u|^{2}+V_{R}(x, t)|u|^{2}\right\}=0 .
\end{gathered}
$$

Next we multiply both sides of (1) by $\bar{u}_{t}$ and take the real parts. Then

$$
\begin{gathered}
-\operatorname{Re}\left\{\nabla \cdot\left(\nabla u \bar{u}_{t}\right)\right\}+\frac{1}{2} \partial_{t}\left\{|\nabla u|^{2}+V_{R}(x, t)|u|^{2}\right\}-\frac{1}{2} \partial_{t} V_{R}(x, t)|u|^{2} \\
-V_{I}(x, t) \operatorname{Im}\left(u \bar{u}_{t}\right)=0 .
\end{gathered}
$$

Getting together these equations, we have

$$
\begin{gathered}
\frac{1}{2} \partial_{t}\left\{|\nabla u|^{2}+V_{R}(x, t)|u|^{2}\right\}-\operatorname{Re}\left[\nabla \cdot\left\{V_{I}(x, t)(\nabla u) \bar{u}+(\nabla u) \bar{u}_{t}\right\}\right] \\
+\operatorname{Re}\left[\left(\nabla V_{I}(x, t) \cdot \nabla u\right) \bar{u}\right]-\frac{1}{2} \partial_{t} V_{R}(x, t)|u|^{2} \\
+V_{I}(x, t)\left\{|\nabla u|^{2}+V_{R}(x, t)|u|^{2}\right\}=0 .
\end{gathered}
$$

Thus, integrating it on $\mathbf{R}^{n} \times(0, t)$ gives the desired identity.

Remark. Let $u_{j}(j=1,2, \ldots)$ be the solution of the modified equation

$$
u_{j}(t)=U_{0}(t)\left(h_{j} * f\right)+i \int_{0}^{t} U_{0}(t-\tau)\left\{h_{j} * V(\cdot, \tau)\left(h_{j} * u(\tau)\right)\right\} d \tau,
$$

where $h_{j}(x) \in C_{0}^{\infty}$ is a series of functions satisfying $h_{j} \rightarrow \delta$ (delta function) as $j \rightarrow \infty$, and $h * g$ means the convolution of $h$ and $g$. Then as is proved in Ginibre-Velo [1] (cf., also Mochizuki-Motai [10]), $u_{j}(t) \rightarrow u(t)$ in $C\left(\mathbf{R}: L^{2}\right.$ ) if $f \in L^{2}$ and $V(x, t)$ satisfies conditions of (i). Moreover, $u_{j}(t) \rightarrow u(t)$ in $C\left(\mathbf{R}: H^{1}\right)$ if $f \in H^{1}$ and $V(x, t)$ satisfies the conditions of (ii).

We shall show that $L^{2}$-decay of solutions occurs under the following condition.

(A1) $V(x, t)$ satisfies

$$
\begin{gathered}
V_{I}(x, t) \geq \phi(|x|+t), \\
\left|\nabla V_{I}(x, t)\right|+\partial_{t} V_{R}(x, t) \leq C_{1} V_{I}(x, t)+\eta(t),
\end{gathered}
$$


where $\phi(\sigma)$ is a positive, bounded continuous function of $\sigma \geq 0$ such that

$$
\int_{0}^{\infty} \phi(\sigma) d \sigma=\infty
$$

$C_{1}$ is a positive constant and $\eta(t)$ is a positive $L^{1}$ function of $t \geq 0$.

Note that potential (6) with (7) satisfies the above condition. In fact, we have

$$
\begin{gathered}
\operatorname{Im} c(1+|x|)^{-\alpha}(1+t)^{-\beta} \geq \operatorname{Im} c(1+|x|+t)^{-\alpha-\beta} \\
\left|\nabla V_{I}(x, t)\right|+\partial_{t} V_{R}(x, t) \leq\left\{\alpha(1+|x|)^{-1}-\beta \frac{\operatorname{Re} c}{\operatorname{Im} c}(1+t)^{-1}\right\} V_{I}(x, t) .
\end{gathered}
$$

So, $(A 1)$ is satisfied with $\phi(\sigma)=\operatorname{Im} c(1+\sigma)^{-\alpha-\beta}, C_{1}=\alpha+\beta \frac{|\operatorname{Re} c|}{\operatorname{Im} c}$ and $\eta(t) \equiv$ 0 .

Lemma 2. Under $(A 1)$, there exists $C_{2}>0$ such that

$$
\|\nabla u(t)\|^{2}+\int_{0}^{t} \int V_{I}(x, t)|\nabla u|^{2} d x d t \leq C_{2}\|f\|_{H^{1}}^{2} \quad \text { for any } t>0 .
$$

Proof. Since $V_{R}(x, t)$ is bounded, it follows from Lemma 1 (i) that

$$
\frac{1}{2} \int\left|V _ { R } ( x , t ) \left\|\left.u\right|^{2} d x+\int_{0}^{t} V_{I}(x, t)\left|V_{R}(x, t)\left\|\left.u\right|^{2} d x d t \leq C\right\| f \|^{2} .\right.\right.\right.
$$

On the other hand, by the second inequality of $(A 1)$ and Lemma 1 (i) we have for any $0<\epsilon<1$,

$$
\begin{aligned}
& \int_{0}^{t} \int\left[V_{I}(x, t)|\nabla u|^{2}+\operatorname{Re}\left\{\left(\nabla V_{I}(x, t) \cdot \nabla u\right) \bar{u}\right\}-\frac{1}{2} \partial_{t} V_{R}(x, t)|u|^{2}\right] d x d t \\
\geq & \int_{0}^{t} \int\left[\left\{(1-\epsilon) V_{I}(x, t)-\epsilon \eta(t)\right\}|\nabla u|^{2}-C_{\epsilon}\left\{C_{1} V_{I}(x, t)+\eta(t)\right\}|u|^{2}\right] d x d t \\
\geq & \int_{0}^{t} \int\left\{(1-\epsilon) V_{I}(x, t)-\eta(t)\right\}|\nabla u|^{2} d x d t-C_{\epsilon}\left(\frac{1}{2} C_{1}+\|\eta\|_{L^{1}}\right)\|f\|^{2} .
\end{aligned}
$$

These inequalities and the identity of Lemma 1 (ii) show

$$
\|\nabla u(t)\|^{2}+\int_{0}^{t} \int\left\{(1-\epsilon) V_{I}(x, t)-\eta(t)\right\}|\nabla u|^{2} d x d t \leq C\|f\|_{H^{1}}^{2} .
$$

In this inequality, we first apply the Gronwall inequality to obtain

$$
\|\nabla u(t)\|^{2} \leq C\left(\|\eta\|_{L^{1}}\right)\|f\|_{H^{1}}^{2} .
$$


Then we have

$$
\int_{0}^{t} \int \eta(t)|\nabla u(t)|^{2} d x d t \leq C\left(\|\eta\|_{L^{1}}\right)\|\eta\|_{L^{1}}\|f\|_{H^{1}}^{2},
$$

and the assertion of the lemma is concluded.

Theorem 1. Assume $(A 1)$. Let $f \in H^{1}$ and also $\sqrt{\varphi(r)} f \in L^{2}$, where $\varphi(\sigma)=\int_{0}^{\sigma} \phi(s) d s+1$ and $r=|x|$. Then

$$
\begin{gathered}
\frac{1}{2}\|\sqrt{\varphi(\cdot+t)} u(t)\|^{2}+\int_{0}^{t} \int \varphi(r+t) V_{I}(x, t)|u|^{2} d x d t \\
\leq \frac{1}{2}\|\sqrt{\varphi(\cdot)} f\|^{2}+2\left(1+C_{2}\right)\|f\|_{H^{1}}^{2}
\end{gathered}
$$

for any $t>0 . \varphi(\sigma)$ being increasing to $\infty$ as $\sigma \rightarrow \infty$, this implies

$$
\|u(t)\|^{2} \leq \varphi(t)^{-1}\left\{\|\sqrt{\varphi(\cdot)} f\|^{2}+2\left(1+C_{2}\right)\|f\|_{H^{1}}^{2}\right\} \rightarrow 0 \text { as } t \rightarrow \infty .
$$

Proof. We multiply by $\varphi(r+t)$ on both sides of (11) and integrate over $\mathbf{R}^{n} \times(0, t)$. Since $\varphi(r)=O(r)$ as $r \rightarrow \infty$, there exists a sequence $R_{k} \rightarrow \infty$ $(k \rightarrow \infty)$ such that

$$
\lim _{k \rightarrow \infty} \operatorname{Im} \int_{0}^{t} \int_{|x|=R_{k}} \varphi\left(\partial_{r} u\right) \bar{u} d S d t=0,
$$

and it follows that

$$
\begin{gathered}
\frac{1}{2}\|\sqrt{\varphi(\cdot+t)} u(t)\|^{2}+\int_{0}^{t} \int\left\{-\frac{1}{2} \phi|u|^{2}+\operatorname{Im}\left(\phi u_{r} \bar{u}\right)+\varphi V_{I}|u|^{2}\right\} d x d \tau \\
=\frac{1}{2}\|\sqrt{\varphi(\cdot)} f\|^{2} .
\end{gathered}
$$

By means of the first inequality of (A1), this and Lemmas 1 (i) and 2 show the theorem.

Next, in order to treat $L^{2}$ nondecay of solutions, we require in contrast to $(A 1)$ the folowing condition.

(A2) $V(x, t)$ satisifes

$$
V_{I}(x, t) \geq 0, \quad|V(x, t)| \leq C_{3} V_{I}(x, t)+\eta(t)
$$

and also

$$
|V(x, t)| \leq \xi(x)+\eta_{1}(t),
$$


where $C_{3}$ is a positive constant, $\eta(t)$ and $\eta_{1}(t)$ are positive $L^{1}$ function of $t>0$ and $\xi(x)$ is a positve function of $x \in \mathbf{R}^{n}$ such that

$$
\xi(x) \in L^{q}\left(\mathbf{R}^{n}\right), \quad \text { for some } 1 \leq q<n .
$$

Note that potential (6) with (8) satisfies this condition. In fact, it follows from the Young inequality that

$$
(1+r)^{-\alpha}(1+t)^{-\beta} \leq \frac{\alpha}{\alpha+\beta}(1+r)^{-\alpha-\beta}+\frac{\beta}{\alpha+\beta}(1+t)^{-\alpha-\beta} .
$$

Since $\alpha+\beta>1$, we can choose $\xi(x)=\epsilon(1+r)^{-\alpha-\beta}$ for $\frac{n}{\alpha+\beta}<q<n$, where $\epsilon$ is any positive constant if $\beta>0$ and $\epsilon=|c|$ if $\beta=0$.

We use the following well known property of free solutions.

Lemma 3. Let $2 \leq p \leq \infty$ and put $\frac{1}{p^{\prime}}=1-\frac{1}{p}$. Let $u_{0}(t)$ be the solution of the free equation (3) with initial condition

$$
u_{0}(x, 0)=f_{0} \in L^{p^{\prime}} .
$$

Then we have

$$
\left\|u_{0}(t)\right\|_{L^{p}} \leq(4 \pi|t|)^{n / p-n / 2}\left\|f_{0}\right\|_{L^{p^{\prime}}} .
$$

Theorem 2. Assume (A2). Then for each $0 \neq f \in L^{2} \cap L^{2 q /(q+1)}$, there exists $s_{0}>0$ such that for all $s>s_{0}$,

$$
U(t, 0)\left[U(0, s) U_{0}(s) f\right]=U(t, s) U_{0}(s) f \nrightarrow 0 \quad \text { as } t \rightarrow \infty .
$$

Proof. Let $u(t)$ and $u_{0}(t)$ be nontrivial $L^{2}$-solutions of (1) and (3), respectively. Then

$$
i \partial_{t}\left(u(t), u_{0}(t)\right)=\left(\Delta u(t)-V u(t), u_{0}(t)\right)-\left(u(t), \Delta u_{0}(t)\right),
$$

where $(\cdot, \cdot)$ is the innerproduct of $L^{2}$. Integrating both sides over $[s, t]$, we have

$$
\left(u(t), u_{0}(t)\right)-\left(u(s), u_{0}(s)\right)-i \int_{s}^{t}\left(V u(\tau), u_{0}(\tau)\right) d \tau=0 .
$$

By the Schwarz inequality

$$
\begin{aligned}
& \left|\left(u(t), u_{0}(t)\right)-\left(u(s), u_{0}(s)\right)\right| \\
& \leq\left\{\int_{s}^{t} \int|V||u|^{2} d x d \tau\right\}^{1 / 2}\left\{\int_{s}^{t} \int|V|\left|u_{0}\right|^{2} d x d \tau\right\}^{1 / 2} .
\end{aligned}
$$


The second inequality of (A2) and Lemma 1 (i) show

$$
\begin{gathered}
\int_{s}^{t} \int|V||u|^{2} d x d \tau \leq \int_{s}^{t} \int\left\{C_{3} V_{I}(x, \tau)+\eta(\tau)\right\}|u|^{2} d x d \tau \\
\leq\left(\frac{C_{3}}{2}+\int_{s}^{t} \eta(\tau) d \tau\right)\|u(s)\|^{2}
\end{gathered}
$$

On the other hand, the third inequality of $(A 2)$ combined with the Hölder inequality shows

(13) $\int_{s}^{t} \int\left|V\left\|\left.u_{0}(\tau)\right|^{2} d x d \tau \leq\right\| \xi\left\|_{L^{q}} \int_{s}^{t}\right\| u_{0}(\tau)\left\|_{L^{2 q^{\prime}}}^{2} d \tau+\int_{s}^{t} \eta(\tau) d \tau\right\| u_{0}(s) \|^{2}\right.$.

Thus, it follows from Lemma 3 that

(14) $\left|\left(u(t), u_{0}(t)\right)-\left(u(s), u_{0}(s)\right)\right| \leq\left(\frac{C_{3}}{2}+\int_{s}^{t} \eta(\tau) d \tau\right)^{1 / 2}\|u(s)\| \times$

$$
\times\left\{C_{4}^{2}\|\xi\|_{L^{q}} \int_{s}^{t} \tau^{-n / q} d \tau\left\|u_{0}(0)\right\|_{L^{2 q /(q+1)}}^{2}+\int_{s}^{t} \eta_{1}(\tau) d \tau\left\|u_{0}(0)\right\|^{2}\right\}^{1 / 2},
$$

where we have used the equalities

$$
2\left(\frac{n}{2}-\frac{n}{2 q^{\prime}}\right)=\frac{n}{q}, \quad 1-\frac{1}{2 q^{\prime}}=\frac{q+1}{2 q} .
$$

Now, for every nonzero $f_{0} \in L^{2} \cap L^{2 q /(q+1)}$, let $u_{0}(t)=U_{0}(t) f_{0}$ and

$$
u(t)=U(t, s) U_{0}(s) f_{0}=U(t, 0)\left\{U(0, s) U_{0}(s) f_{0}\right\}
$$

We can show that this $u(t)$ does not decay as $t \rightarrow \infty$. In fact, contrary to the conclusion, assume that $\|u(t)\| \rightarrow 0$ as $t \rightarrow \infty$. Then letting $t \rightarrow \infty$ in (14), we obtain

$$
\begin{gathered}
\left\|U_{0}(s) f_{0}\right\| \leq\left(\frac{C_{3}}{2}+\int_{s}^{\infty} \eta(\tau) d \tau\right)^{1 / 2} \times \\
\times\left\{C_{4}^{2}\|\xi\|_{L^{q}} \int_{s}^{\infty} \tau^{-n / q} d \tau\left\|f_{0}\right\|_{L^{2 q /(q+1)}}^{2}+\int_{s}^{\infty} \eta_{1}(\tau) d \tau\left\|f_{0}\right\|^{2}\right\}^{1 / 2},
\end{gathered}
$$

Since $\left\|U_{0}(s) f_{0}\right\|$ is independent of $s$, this leads to a contradiction if $s$ is chosen sufficiently large. 


\section{$\S 3 . \quad$ Space-time $L^{p}-L^{q}$ Estimates}

In this section we first summarize space-time $L^{p}-L^{q}$ estimates of free solutions, and then use it to obtain similar estimates of perturbed solutions.

Lemma 4. Let $n \geq 3$ and let $\frac{n-2}{2 n} \leq \frac{1}{p} \leq \frac{1}{2}$ and $\frac{1}{r}=\frac{n}{2}\left(\frac{1}{2}-\frac{1}{p}\right)$. Then there exists $C_{5}>0$ such that

$$
\left\|\int_{0}^{t} U_{0}(t-\tau) h(\tau) d \tau\right\|_{L^{r}\left(\mathbf{R}_{ \pm} ; L^{p}\right)} \leq C_{5}\|h\|_{L^{r^{\prime}}\left(\mathbf{R}_{ \pm} ; L^{p^{\prime}}\right)} .
$$

As is well known this lemma is a direct from Lemma 3 if $\frac{1}{p}>\frac{n-2}{2 n}$. At the end point $\frac{1}{p}=\frac{n-2}{2 n}$, it is due to Keer-Tao [6].

As a corollary of this lemma we have the following

Lemma 5. Let $n, p$ and $r$ be as in Lemma 4. Then

(i) For any $t \in \mathbf{R}_{ \pm}$,

$$
\left\|\int_{0}^{t} U_{0}(-\tau) h(\tau) d \tau\right\| \leq \sqrt{2 C_{5}}\|h\|_{L^{r^{\prime}}\left(\mathbf{R}_{ \pm} ; L^{p^{\prime}}\right)} .
$$

(ii) For $f_{0} \in L^{2}$, we have $U_{0}(t) f_{0} \in L^{r}\left(\mathbf{R}_{ \pm} ; L^{p}\right)$ and

$$
\left\|U_{0}(\cdot) f_{0}\right\|_{L^{r}\left(\mathbf{R}_{ \pm} ; L^{p}\right)} \leq \sqrt{2 C_{5}}\left\|f_{0}\right\| .
$$

Now, we return to the perturbed problem. We obtain similar estimates of perturbed solutions requiring the following condition on $V(x, t)$.

(A3) $V(x, t)$ satisfies

$$
V(x, t) \in L^{\nu}\left(\mathbf{R} ; L^{q}\right)
$$

where

$$
0 \leq \frac{1}{q} \leq \frac{2}{n} \quad \text { and } \quad \frac{1}{\nu}=1-\frac{n}{2 q}
$$

Moreover, $V(x, t)$ satisfies the smallness condition

$$
C_{5}\|V\|_{L^{\infty}\left(\mathbf{R}_{ \pm} ; L^{n / 2}\right)}<1 \quad \text { when } \quad \nu=\infty
$$

where $C_{5}$ is a constant given in Lemma 4 .

Note that potential (6) with (9) satisfies this condition (A3) if we choose

$$
\frac{1}{q}=0 \text { when } \alpha=0, \quad \frac{1}{q}=\frac{2}{n} \text { when } \beta=0 \quad \text { and }
$$




$$
\frac{\max \{0,2(1-\beta)\}}{n}<\frac{1}{q}<\frac{\min \{\alpha, 2\}}{n} \text { when } \alpha, \beta>0 .
$$

For $1 \leq \gamma, \mu \leq \infty$ and $\pm s \geq 0$, we put

$$
Y_{ \pm, s}^{\gamma, \mu}=L^{\gamma}\left(\mathbf{R}_{ \pm, s} ; L^{\mu}\right)
$$

where $\mathbf{R}_{+}, s=[s, \infty)$ for $s \geq 0$ and $\mathbf{R}_{-}, s=(-\infty, s]$ for $s \leq 0$. The space $Y_{ \pm, 0}^{\gamma, \mu}=L^{\gamma}\left(\mathbf{R}_{ \pm} ; L^{\mu}\right)$ is already used in this Section. By $(A 3)$ we have $V(x, t) \in$ $Y_{ \pm, s}^{\nu, q}$ for any $\pm s \geq 0$. Moreover, as we see from (15), there exists $\pm s \geq 0$ such that

$$
C_{5}\|V\|_{Y_{ \pm, s}^{\nu, q}}<1 .
$$

In the following we fix such an $s$, and choose the pair $\{p, r\}$, related to $\{q, \nu\}$, as follows:

$$
\frac{1}{p}=\frac{1}{2}\left(1-\frac{1}{q}\right), \quad \text { and } \quad \frac{1}{r}=\frac{1}{2}\left(1-\frac{1}{\nu}\right) .
$$

As is easily seen, the condition for $\{q, \nu\}$ in $(A 3)$ is equivalent to that for $\{p, r\}$ in Lemma 4.

Theorem 3. Let $n \geq 3$ and assume (A3). Then for each $f \in L^{2}$,

(i) The integral equation

$$
u(t)=U_{0}(t-s) f+i \int_{s}^{t} U_{0}(t-\tau) V(\tau) u(\tau) d \tau
$$

has a unique solution in $u(t) \in Y_{ \pm, s}^{r, p}$.

(ii) This solution belongs to $C\left(\mathbf{R}_{ \pm, s} ; L^{2}\right)$ and coincides with $U(t, s) f$. Moreover, we have

$$
\|u\|_{Y_{ \pm, s}^{r, p}} \leq \frac{\sqrt{2 C_{5}}}{1-C_{5}\|V\|_{Y_{ \pm, s}^{\nu, q}}}\|f\|
$$

and

$$
\left\|\int_{s}^{t} U_{0}(-\tau) V(\tau) u(\tau) d \tau\right\| \leq \frac{2 C_{5}\|V\|_{Y_{ \pm, s}^{\nu, q}}}{1-C_{5}\|V\|_{Y_{ \pm . s}^{\nu, q}}}\|f\| .
$$

Proof. (i) For $g(t) \in Y_{ \pm, s}^{r, p}$, we put

$$
\Phi_{ \pm, s} g(t)=\int_{s}^{t} U_{0}(t-\tau) V(\tau) g(\tau) d \tau, \quad t \in \mathbf{R}_{ \pm} .
$$

By Lemma 4 we have

$$
\left\|\int_{s}^{t} U_{0}(t-\tau) V(\tau) g(\tau) d \tau\right\|_{Y_{ \pm, s}^{r, p}} \leq C_{5}\|V g\|_{Y_{ \pm, s}^{r^{\prime}, p^{\prime}}} .
$$


Here

$$
\|V g\|_{Y_{ \pm, s}^{r^{\prime}, p^{\prime}}} \leq\|V\|_{Y_{ \pm, s}^{\nu, q}}\|g\|_{Y_{ \pm, s}^{r^{\prime} \nu /\left(\nu-r^{\prime}\right), p^{\prime} q /\left(q-p^{\prime}\right)}}
$$

and (17) implies that $\frac{p^{\prime} q}{q-p^{\prime}}=p$ and $\frac{r^{\prime} \nu}{\nu-r^{\prime}}=r$. Thus, the above inequality proves that

$$
\left\|\Phi_{ \pm, s} g\right\|_{Y_{ \pm, s}^{r, p}} \leq C_{5}\|V\|_{Y_{ \pm, s}^{\nu, q}}\|g\|_{Y_{ \pm, s}^{r, p}}
$$

Now, for $f \in L^{2}$ we define $\left\{u_{k}(t)\right\}$ successively as follows:

$$
u_{0}(t)=U_{0}(t-s) f, \quad u_{k}(t)=u_{0}(t)+i \Phi_{ \pm, s} u_{k-1}(t) .
$$

$u_{0}(t) \in Y_{ \pm, s}^{r, p}$ by Lemma 5 (ii), and hence, each $u_{k}(t) \in Y_{ \pm, s}^{r, p}$ by (20). Moreover, since

$$
\left\|u_{n}-u_{n-1}\right\|_{Y_{ \pm, s}^{r, p}} \leq\left(\|\Phi\|_{\mathcal{B}\left(Y_{ \pm, s}^{r, p}\right)}\right)^{n}\left\|u_{0}\right\|_{Y_{ \pm, s}^{r, p}}
$$

and $\left\|\Phi_{ \pm, s}\right\|_{\mathcal{B}\left(Y_{ \pm, s}^{r, p}\right)}<1$ by (16) and (20), we see that $\left\{u_{n}(t)\right\}$ converges in $Y_{ \pm, s}^{r, p}$ as $n \rightarrow \infty$.

It is obvious that the limit $u=u(t)$ is the desired solution of the integral equation.

(ii) It follows from Lemma 5 (i) that

$$
\left\|\int_{s}^{t} U_{0}(-\tau) V(\tau) u(\tau) d \tau\right\| \leq \sqrt{2 C_{5}}\|V u\|_{Y_{ \pm, s}^{r^{\prime}, p^{\prime}}} \leq \sqrt{2 C_{5}}\|V\|_{Y_{ \pm, s}^{\nu, q}}\|u\|_{Y_{ \pm, s}^{r, p}}
$$

This and the integral equation show that the solution $u(t)$ is in $C\left(\mathbf{R}_{ \pm, s} ; L^{2}\right)$. Since the integral equation has a unique solution in $C\left(\mathbf{R}_{ \pm, s} ; L^{2}\right)$, this $u(t)$ coincides with $U(t, s) f$. Moreover, inequality (18) easily follows from the definition

$$
u(t)=u_{0}(t)+\sum_{k=1}^{\infty}\left\{u_{k}(t)-u_{k-1}(t)\right\}
$$

if we note $\left\|u_{0}\right\|_{Y_{ \pm, s}^{r, p}} \leq \sqrt{2 C_{5}}\|f\|$.

Inequality (19) follows from (18) combined with (21).

\section{$\S 4$. Scattering}

Our results on scattering are summarized in the following theorem.

Theorem 4. Let $n \geq 3$ and assume $(A 3)$. Then

(i) For every $f \in L^{2}$ there exists $f_{0}^{ \pm} \in L^{2}$ such that

$$
\left\|U(t, 0) f-U_{0}(t) f_{0}^{ \pm}\right\|^{2} \rightarrow 0 \quad \text { as } \quad t \rightarrow \pm \infty .
$$


We put

$$
Z^{ \pm}=s-\lim _{t \rightarrow \pm \infty} U_{0}(-t) U(t, 0) .
$$

Then $Z^{ \pm}$defines a nontrivial bounded operator on $L^{2}$.

(ii) If (15) in (A3) is replaced by the stronger condition

$$
3 C_{5}\|V\|_{L^{\infty}\left(\mathbf{R}_{ \pm} ; L^{n / 2}\right)}<1 \quad \text { when } \quad \nu=\infty
$$

then $Z^{ \pm}$gives a bijection on $L^{2}$. Thus, the scattering operator

$$
S=Z^{+}\left(Z^{-}\right)^{-1}: \quad f_{0}^{-} \rightarrow f_{0}^{+}
$$

is well defined and also gives a bijection on $L^{2}$.

Proof. (i) We put $u(t)=U(t, s) f$ and $u_{0}(t)=U_{0}(t-s) f_{0}$. Then as in the proof of Theorem 2 we have

$$
\left(u(t), u_{0}(t)\right)-\left(u(\sigma), u_{0}(\sigma)\right)-i \int_{\sigma}^{t}\left(V(\tau) u(\tau), u_{0}(\tau)\right) d \tau=0
$$

for any $\sigma, t \in \mathbf{R}_{ \pm, s}$. It follows from $(A 3)$ and Lemma 5 that

$$
\begin{aligned}
\left.\left.\left|\int_{\sigma}^{t} \int\right| V|| u_{0}\right|^{2} d x d \tau\right|^{1 / 2} & \leq\|V\|_{Y_{ \pm, s}^{\nu, q}}^{1 / 2}\left\|u_{0}\right\|_{Y_{ \pm, s}^{2 \nu^{\prime}, 2 q^{\prime}}} \\
& \leq \sqrt{2 C_{5}}\|V\|_{Y_{ \pm, s}^{\nu, q}}^{1 / 2}\left\|f_{0}\right\|
\end{aligned}
$$

where we have used the equalities

$$
\frac{1}{2 q^{\prime}}=\frac{1}{2}\left(1-\frac{1}{q}\right)=\frac{1}{p}, \quad \frac{1}{2 \nu^{\prime}}=\frac{1}{r} .
$$

On the other hand, by $(A 3)$ and Theorem 3 (ii) we similarly have

$$
\left.\left.\left|\int_{\sigma}^{t} \int\right| V|| u\right|^{2} d x d \tau\right|^{1 / 2} \leq \frac{\sqrt{2 C_{5}}\|V\|_{Y_{ \pm, s}^{\nu, q}}^{1 / 2}}{1-C_{5}\|V\|_{Y_{ \pm, s}^{\nu, q}}}\|f\| .
$$

Now we have from (23) and (24)

$$
\begin{aligned}
& \left|\left(U_{0}(s-t) U(t, s) f-U_{0}(s-\sigma) U(\sigma, s) f, f_{0}\right)\right| \\
& \quad \leq\left|\int_{\sigma}^{ \pm \infty} \int\right| V(\tau)\left\|\left.\left.u(\tau)\right|^{2} d \tau\right|^{1 / 2} \sqrt{2 C_{5}}\right\| V\left\|_{Y_{ \pm, s}^{\nu, q}}^{1 / 2}\right\| f_{0} \| .
\end{aligned}
$$


Since

$$
\left.\left.\left|\int_{\sigma}^{ \pm \infty} \int\right| V|| u\right|^{2} d x d \tau\right|^{1 / 2} \rightarrow 0 \quad \text { as } \sigma \rightarrow \pm \infty
$$

this shows the existence of the strong limit

$$
Z^{ \pm}(s)=s-\lim _{t \rightarrow \pm \infty} U_{0}(s-t) U(t, s)
$$

in $L^{2}$, and we also have

$$
Z^{ \pm}=s-\lim _{t \rightarrow \pm \infty} U_{0}(-t) U(t, 0)=U_{0}(-s) Z^{ \pm}(s) U(s, 0) .
$$

The nontriviality of $Z^{ \pm}$is easily verified if we use (18) and follow the proof of Theorem 2.

(ii) To verify the assertions, we have only to show that $Z^{ \pm}(s)$ is a bijection on $L^{2}$. For this aim we use the following inequality due to (23), (24) and (25).

$$
\begin{gathered}
\left|\left(U_{0}(s-t) U(t, s) f-U_{0}(s-\sigma) U(\sigma, s) f, f_{0}\right)\right| \\
\leq \frac{2 C_{5}\|V\|_{Y_{ \pm, s}^{\nu, q}}}{1-C_{5}\|V\|_{Y_{ \pm, s}^{\nu, q}}}\|f\|\left\|f_{0}\right\| .
\end{gathered}
$$

We put $\sigma=s$ and let $t \rightarrow \pm \infty$. Then it follows from this inequality that

$$
\left|\left(\left\{Z_{ \pm}(s)-I\right\} f, f_{0}\right)\right| \leq \frac{2 C_{5}\|V\|_{Y_{ \pm, s}^{\nu, q}}}{1-C_{5}\|V\|_{Y_{ \pm, s}^{\nu, q}}}\|f\|\left\|f_{0}\right\| .
$$

Since

$$
\frac{2 C_{5}\|V\|_{Y_{ \pm, s}^{\nu, q}}}{1-C_{5}\|V\|_{Y_{ \pm, s}^{\nu, q}}}<1
$$

this implies $\left\|Z_{ \pm}-I\right\|_{\mathcal{B}\left(L^{2}\right)}<1$ and the proof is completed.

\section{References}

[1] J. Ginibre and G. Velo, On a class of nonlinear Schrödinger equation I, J. Functional Analysis 32 (1972), 1-32.

[2] J. S. Howland, Stationary scattering theory for time dependent Hamiltonians, Math. Ann. 207 (1974), 315-335.

[3] A. Jensen, Results in $L^{p}\left(\mathbf{R}^{d}\right)$ for the Schrödinger equation with time-dependent potential, Math. Ann. 299 (1994), 117-125.

[4] T. Kato, Wave operators and similarity for some non-selfadjoint operators, Math. Ann. $162(1965 / 1966), 258-279$. 
[5] T. Kato and K. Yajima, Some examples of smooth operators and the associated smoothing effect, Rev. Math. Phys. 1 (1989), no. 4, 481-496.

[6] M. Keel and R. Tao, Endpoint Strichartz estimates, Ameri. J. Math. 120 (1998), no. 5, 955-980.

[7] H. Kitada and K. Yajima, A scattering theory for time-dependent long-range potentials, Duke Math. J. 49 (1982), no. 2, 341-376.

[8] K. Mochizuki, Eigenfunction expansions associated with the Schrödinger operator with a complex potential and the scattering inverse problem, Proc. Japan Acad. 43 (1967), 638-643.

[9] Math. 31 (2007), no. 2, to appear.

[10] K. Mochizuki and T. Motai, The scattering theory for the nonlinear wave equation with small data. II, Publ. Res. Inst. Math. Sci. 23 (1987), no. 5, 771-790.

[11] K. Mochizuki and H. Nakazawa, Energy decay and asymptotic behavior of solutions to the wave equation with linear dissipation, Publ. Res. Inst. Math. Sci. 32 (1996), no. 3, 401-414.

[12] D. R. Yafaev, On the violation of the unitarity in time dependent potential scattering, Soviet Math. Dokl., 19 (1973), 1517-1521.

[13] K. Yajima, Scattering theory for Schrödinger equations with potentials periodic in time, J. Math. Soc. Japan 29 (1977), no. 4, 729-743. 